International Journal of Pure and Applied Mathematics

Volume 89 No. 5 2013, 659-665

ISSN: 1311-8080 (printed version); ISSN: 1314-3395 (on-line version)

url: http://www.ijpam.eu

doi: http://dx.doi.org/10.12732/ijpam.v89i5.2

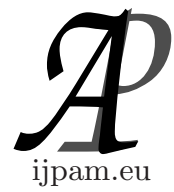

\title{
CUBIC KU-SUBALGEBRAS
}

\author{
Muhammad Akram ${ }^{1}$, Naveed Yaqoob ${ }^{2}$, Muhammad Gulistan ${ }^{3}$ \\ ${ }^{1}$ Department of Mathematics \\ University of Gujrat \\ Gujrat, PAKISTAN \\ ${ }^{2}$ Department of Mathematics \\ COMSATS Institute of Information Technology \\ Abbottabad, PAKISTAN \\ ${ }^{3}$ Department of Mathematics \\ Hazara University \\ Mansehra, PAKISTAN
}

Abstract: In this paper, we study some new properties of cubic KU-subalgebras.

AMS Subject Classification: 06F35, 03G25, 08A72

Key Words: KU-algebras, cubic sets, cubic KU-subalgebras

\section{Introduction}

Prabpayak and Leerawat [3] introduced a new algebraic structure which is called KU-algebra. They introduced the concept of homomorphisms of KU-algebras and investigated some related properties in [4]. Mostafa et al. [2] introduced the notion of fuzzy KU-ideals of KU-algebras.

Jun et al. [1] introduced the notion of cubic sub-algebras and ideals in BCK/BCI-algebras. They discussed relationship between a cubic subalgebra and a cubic ideal.

Received: June 27, 2013

(C) 2013 Academic Publications, Ltd.

${ }^{\S}$ Correspondence author 
In this paper, we provided some new properties of cubic KU-subalgebras.

\section{Preliminaries and Basic Definitions}

In this section, we will recall some definitions of KU-algebra and cubic sets.

Definition 1. By a KU-algebra we mean an algebra $(X, *, 0)$ of type $(2,0)$ with a single binary operation $*$ that satisfies the following identities: for any $x, y, z \in X$,

$$
\begin{aligned}
& (\mathrm{ku} 1)(x * y) *[(y * z) *(x * z)]=0, \\
& (\mathrm{ku} 2) x * 0=0, \\
& (\mathrm{ku} 3) 0 * x=x, \\
& (\mathrm{ku} 4) x * y=0=y * x \text { implies } x=y .
\end{aligned}
$$

In a KU-algebra, the identity $z * z=0$ is true [2].

Definition 2. [4] A subset $S$ of KU-algebra $X$ is called a KU-subalgebra of $X$ if $x * y \in S$, whenever $x, y \in S$.

An interval number is $\widetilde{a}=\left[a^{-}, a^{+}\right]$, where $0 \leq a^{-} \leq a^{+} \leq 1$. Let $D[0,1]$ denote the family of all closed subintervals of $[0,1]$, i.e.,

$$
D[0,1]=\left\{\widetilde{a}=\left[a^{-}, a^{+}\right]: a^{-} \leq a^{+}, \text {for } a^{-}, a^{+} \in I\right\}
$$

We define the operations " $\succeq ", " \preceq ", "==$, "rmin" and "rmax" in case of two elements in $D[0,1]$. We consider two elements $\widetilde{a}=\left[a^{-}, a^{+}\right]$and $\widetilde{b}=\left[b^{-}, b^{+}\right]$ in $D[0,1]$. Then:

(1) $\widetilde{a} \succeq \widetilde{b}$ if and only if $a^{-} \geq b^{-}$and $a^{+} \geq b^{+}$,

(2) $\widetilde{a} \preceq \widetilde{b}$ if and only if $a^{-} \leq b^{-}$and $a^{+} \leq b^{+}$,

(3) $\operatorname{rmin}\{\widetilde{a}, \widetilde{b}\}=\left[\min \left\{a^{-}, b^{-}\right\}, \min \left\{a^{+}, b^{+}\right\}\right]$.

Definition 3. [1] Cubic sets defined on a non-empty set $X$ as object having the form $\Xi=\left\{\left\langle x, \widetilde{\delta}_{\Xi}(x), \theta_{\Xi}(x)\right\rangle: x \in X\right\}$, which is briefly denoted by $\Xi=\left\langle\widetilde{\delta}_{\Xi}, \theta_{\Xi}\right\rangle$, where the functions $\widetilde{\delta}_{\Xi}: X \rightarrow D[0,1]$ and $\theta_{\Xi}: X \rightarrow[0,1]$. 


\section{Cubic KU-Subalgebras}

In this section, we will discuss some new properties of cubic KU-subalgebras.

Definition 4. Let $X$ be a KU-algebra. A cubic subset $\Xi=\left\langle\widetilde{\delta}_{\Xi}, \theta_{\Xi}\right\rangle$ in $X$ is called a cubic KU-subalgebra of $X$ if

$$
\widetilde{\delta}_{\Xi}(x * y) \succeq r \min \left\{\widetilde{\delta}_{\Xi}(x), \widetilde{\delta}_{\Xi}(y)\right\}
$$

and

$$
\theta_{\Xi}(x * y) \leq \max \left\{\theta_{\Xi}(x), \theta_{\Xi}(y)\right\}
$$

for all $x, y \in X$.

Proposition 5. If $\Xi=\left\langle\widetilde{\delta}_{\Xi}, \theta_{\Xi}\right\rangle$ is a cubic KU-subalgebra of $X$, then for all $x \in X, \widetilde{\delta}_{\Xi}(0) \succeq \widetilde{\delta}_{\Xi}(x)$ and $\theta_{\Xi}(0) \leq \theta_{\Xi}(x)$.

Proof. The proof is straightforward.

Theorem 6. Let $\Xi=\left\langle\widetilde{\delta}_{\Xi}, \theta_{\Xi}\right\rangle$ be a cubic KU-subalgebra of $X$. If there exists a sequence $x_{n}$ in $X$ such that $\lim _{n \rightarrow \infty} \widetilde{\delta}_{\Xi}\left(x_{n}\right)=[1,1]$ and $\lim _{n \rightarrow \infty} \theta_{\Xi}\left(x_{n}\right)=0$, then $\widetilde{\delta}_{\Xi}(0)=[1,1]$ and $\theta_{\Xi}(0)=0$.

Proof. Let $\Xi=\left\langle\widetilde{\delta}_{\Xi}, \theta_{\Xi}\right\rangle$ be a cubic KU-subalgebra of $X$. By Proposition $5, \widetilde{\delta}_{\Xi}(0) \succeq \widetilde{\delta}_{\Xi}(x)$ for all $x \in X$, therefore $\widetilde{\delta}_{\Xi}(0) \succeq \widetilde{\delta}_{\Xi}\left(x_{n}\right)$ for positive integer $n$. Consider, $[1,1] \succeq \widetilde{\delta}_{\Xi}(0) \succeq \lim _{n \rightarrow \infty} \widetilde{\delta}_{\Xi}\left(x_{n}\right)=[1,1]$. Hence, $\widetilde{\delta}_{\Xi}(0)=[1,1]$.

Also, by Proposition $5, \theta_{\Xi}(0) \leq \theta_{\Xi}(x)$ for all $x \in X$, thus $\theta_{\Xi}(0) \leq \theta_{\Xi}\left(x_{n}\right)$ for positive integer $n$. Now, $0 \leq \theta_{\Xi}(0) \leq \lim _{n \rightarrow \infty} \theta_{\Xi}\left(x_{n}\right)=0$. Hence, $\theta_{\Xi}(0)=0$.

For any elements $x$ and $y$ of $X$, let us write

$$
\prod^{m} x * y \text { for }(\ldots((x * x) * x) \ldots) * y
$$

where $x$ appears $m$ times. Here $m$ is a natural number.

Theorem 7. Let $\Xi=\left\langle\widetilde{\delta}_{\Xi}, \theta_{\Xi}\right\rangle$ be a cubic KU-subalgebra of $X$ and let $m \in N$ ( $N$ be the set of natural numbers). Then

(i) $\widetilde{\delta}_{\Xi}\left(\stackrel{m}{\prod} x * x\right) \succeq \widetilde{\delta}_{\Xi}(x)$ and $\theta_{\Xi}\left(\prod^{m} x * x\right) \leq \theta_{\Xi}(x)$, for any odd number $m$,

(ii) $\widetilde{\delta}_{\Xi}\left(\prod^{m} x * x\right)=\widetilde{\delta}_{\Xi}(x)$ and $\theta_{\Xi}\left(\prod^{m} x * x\right)=\theta_{\Xi}(x)$, for any even number $m$. 
Proof. (i) Let $\Xi=\left\langle\widetilde{\delta}_{\Xi}, \theta_{\Xi}\right\rangle$ be a cubic KU-subalgebra of $X$ and let $x \in X$. Assume that $m$ is odd. Then $m=2 q-1$ for some positive integer $q$. Now we prove the theorem by induction. Now by Proposition $5, \widetilde{\delta}_{\Xi}(x * x)=\widetilde{\delta}_{\Xi}(0) \succeq$ $\widetilde{\delta}_{\Xi}(x)$ and $\theta_{\Xi}(x * x)=\theta_{\Xi}(0) \leq \theta_{\Xi}(x)$. Suppose that $\widetilde{\delta}_{\Xi}\left(\prod^{2 q-1} x * x\right) \succeq \widetilde{\delta}_{\Xi}(x)$ and $\theta_{\Xi}\left(\prod^{2 q-1} x * x\right) \leq \theta_{\Xi}(x)$. Then by assumption,

$$
\begin{aligned}
\widetilde{\delta}_{\Xi}\left(\prod^{2(q+1)-1} x * x\right) & =\widetilde{\delta}_{\Xi}\left(\prod^{2 q+1} x * x\right) \\
& =\widetilde{\delta}_{\Xi}\left(\prod^{2 q-1} x *((x * x) * x)\right) \\
& =\widetilde{\delta}_{\Xi}\left(\prod^{2 q-1} x *(0 * x)\right) \\
& =\widetilde{\delta}_{\Xi}\left(\prod^{2 q-1} x * x\right) \succeq \widetilde{\delta}_{\Xi}(x)
\end{aligned}
$$

and

$$
\begin{aligned}
\theta_{\Xi}\left(\prod^{2(q+1)-1} x * x\right) & =\theta_{\Xi}\left(\prod^{2 q+1} x * x\right) \\
& =\theta_{\Xi}\left(\prod^{2 q-1} x *((x * x) * x)\right) \\
& =\theta_{\Xi}\left(\prod^{2 q-1} x *(0 * x)\right) \\
& =\theta_{\Xi}\left(\prod^{2 q-1} x * x\right) \leq \theta_{\Xi}(x)
\end{aligned}
$$

This completes the proof of (i). The proof of (ii) is similar to (i).

Theorem 8. Let $\mathcal{A}$ be a non-empty subset of $X$ and $\Xi=\left\langle\widetilde{\delta}_{\Xi}, \theta_{\Xi}\right\rangle$ be a cubic subset in $X$ defined by

$$
\widetilde{\delta}_{\Xi}(x)=\left\{\begin{array}{ll}
{\left[\beta_{1}, \beta_{2}\right]} & \text { if } x \in \mathcal{A} \\
{\left[\gamma_{1}, \gamma_{2}\right]} & \text { otherwise }
\end{array} \quad \text { and } \theta_{\Xi}(x)= \begin{cases}\vartheta & \text { if } x \in \mathcal{A} \\
\eta & \text { otherwise }\end{cases}\right.
$$


for all $\left[\beta_{1}, \beta_{2}\right],\left[\gamma_{1}, \gamma_{2}\right] \in D[0,1]$ and $\vartheta, \eta \in[0,1]$ with $\left[\beta_{1}, \beta_{2}\right] \succeq\left[\gamma_{1}, \gamma_{2}\right]$ and $\vartheta \leq \eta$. Then $\Xi=\left\langle\widetilde{\delta}_{\Xi}, \theta_{\Xi}\right\rangle$ is a cubic $K U$-subalgebra of $X$ if and only if $\mathcal{A}$ is a $K U$-subalgebra of $X$.

Proof. Let $\Xi=\left\langle\widetilde{\delta}_{\Xi}, \theta_{\Xi}\right\rangle$ be a cubic KU-subalgebra of $X$. Let $x, y \in X$ be such that $x, y \in \mathcal{A}$. Then

$$
\begin{aligned}
\widetilde{\delta}_{\Xi}(x * y) & \succeq r \min \left\{\widetilde{\delta}_{\Xi}(x), \widetilde{\delta}_{\Xi}(y)\right\} \\
& =r \min \left\{\left[\beta_{1}, \beta_{2}\right],\left[\beta_{1}, \beta_{2}\right]\right\}=\left[\beta_{1}, \beta_{2}\right]
\end{aligned}
$$

and

$$
\theta_{\Xi}(x * y) \leq \max \left\{\theta_{\Xi}(x), \theta_{\Xi}(y)\right\}=\max \{\vartheta, \vartheta\}=\vartheta .
$$

So $x * y \in \mathcal{A}$. Hence $\mathcal{A}$ is a $\mathrm{KU}$-subalgebra of $X$.

Conversely, suppose that $\mathcal{A}$ is a KU-subalgebra of $X$. Let $x, y \in X$. We have two cases here:

Case (i): If $x, y \in \mathcal{A}$ then $x * y \in \mathcal{A}$, thus

$$
\widetilde{\delta}_{\Xi}(x * y)=\left[\beta_{1}, \beta_{2}\right]=r \min \left\{\widetilde{\delta}_{\Xi}(x), \widetilde{\delta}_{\Xi}(y)\right\}
$$

and

$$
\theta_{\Xi}(x * y)=\vartheta=\max \left\{\theta_{\Xi}(x), \theta_{\Xi}(y)\right\} .
$$

Case (ii): If $x \notin \mathcal{A}$ or $y \notin \mathcal{A}$, then

$$
\widetilde{\delta}_{\Xi}(x * y) \succeq\left[\gamma_{1}, \gamma_{2}\right]=r \min \left\{\widetilde{\delta}_{\Xi}(x), \widetilde{\delta}_{\Xi}(y)\right\}
$$

and

$$
\theta_{\Xi}(x * y) \leq \eta=\max \left\{\theta_{\Xi}(x), \theta_{\Xi}(y)\right\} .
$$

Hence, $\Xi=\left\langle\widetilde{\delta}_{\Xi}, \theta_{\Xi}\right\rangle$ is a cubic KU-subalgebra of $X$.

Theorem 9. If every cubic $K U$-subalgebra $\Xi=\left\langle\widetilde{\delta}_{\Xi}, \theta_{\Xi}\right\rangle$ of $X$ has the finite image, then every descending chain of $K U$-subalgebras of $X$ terminates at finite step.

Proof. Consider a strictly descending chain $H_{0} \supset H_{1} \supset H_{2} \cdots$ of KUsubalgebras of $X$ which does not terminate at finite step. Define a cubic set $\Xi=\left\langle\widetilde{\delta}_{\Xi}, \theta_{\Xi}\right\rangle$ in $X$ by

$$
\widetilde{\delta}_{\Xi}(x)= \begin{cases}{\left[\frac{n+1}{n+2}, \frac{n+3}{n+4}\right]} & \text { if } x \in H_{n} \backslash H_{n+1} \\ {[1,1]} & \text { if } x \in \cap_{n=0}^{\infty} H_{n}\end{cases}
$$




$$
\text { and } \theta_{\Xi}(x)= \begin{cases}\frac{n+2}{n+3} & \text { if } x \in H_{n} \backslash H_{n+1} \\ 0 & \text { if } x \in \cap_{n=0}^{\infty} H_{n}\end{cases}
$$

where $n=0,1,2, \ldots$ and $H_{0}$ stands for $X$. Let $x, y \in X$. Assume that $x \in$ $H_{n} \backslash H_{n+1}$ and $y \in H_{k} \backslash H_{k+1}$ for $n=0,1,2, \ldots ; k=0,1,2, \ldots$ We may assume that $n \leq k$. Then obviously $x$ and $y \in H_{n}$, so $x * y \in H_{n}$ because $H_{n}$ is a KU-subalgebra of $X$. Hence,

$$
\begin{aligned}
& \widetilde{\delta}_{\Xi}(x * y) \succeq\left[\frac{n+1}{n+2}, \frac{n+3}{n+4}\right]=\operatorname{rmin}\left\{\widetilde{\delta}_{\Xi}(x), \widetilde{\delta}_{\Xi}(y)\right\} \\
& \theta_{\Xi}(x * y) \leq \frac{n+2}{n+3}=\max \left\{\theta_{\Xi}(x), \theta_{\Xi}(y)\right\} .
\end{aligned}
$$

If $x, y \in \cap_{n=0}^{\infty} H_{n}$, then $x * y \in \cap_{n=0}^{\infty} H_{n}$. Thus

$$
\widetilde{\delta}_{\Xi}(x * y)=[1,1]=r \min \left\{\widetilde{\delta}_{\Xi}(x), \widetilde{\delta}_{\Xi}(y)\right\}
$$

and

$$
\theta_{\Xi}(x * y)=0=\max \left\{\theta_{\Xi}(x), \theta_{\Xi}(y)\right\}
$$

If $x \notin \cap_{n=0}^{\infty} H_{n}$ and $y \in \cap_{n=0}^{\infty} H_{n}$, then there exists a positive integer $p$ such that $x \in H_{p} \backslash H_{p+1}$. It follows that $x * y \in H_{p}$ so that

$$
\begin{aligned}
& \widetilde{\delta}_{\Xi}(x * y) \succeq\left[\frac{p+1}{p+2}, \frac{p+3}{p+4}\right]=\operatorname{rmin}\left\{\widetilde{\delta}_{\Xi}(x), \widetilde{\delta}_{\Xi}(y)\right\} \\
& \theta_{\Xi}(x * y) \leq \frac{p+2}{p+3}=\max \left\{\theta_{\Xi}(x), \theta_{\Xi}(y)\right\} .
\end{aligned}
$$

Finally suppose that $x \in \cap_{n=0}^{\infty} H_{n}$ and $y \notin \cap_{n=0}^{\infty} H_{n}$. Then $y \in H_{q} \backslash H_{q+1}$ for some positive integer $q$. It follows that $x * y \in H_{q}$, and hence

$$
\begin{aligned}
& \widetilde{\delta}_{\Xi}(x * y) \succeq\left[\frac{q+1}{q+2}, \frac{q+3}{q+4}\right]=\operatorname{rmin}\left\{\widetilde{\delta}_{\Xi}(x), \widetilde{\delta}_{\Xi}(y)\right\} \\
& \theta_{\Xi}(x * y) \leq \frac{q+2}{q+3}=\max \left\{\theta_{\Xi}(x), \theta_{\Xi}(y)\right\}
\end{aligned}
$$

This proves that $\Xi=\left\langle\widetilde{\delta}_{\Xi}, \theta_{\Xi}\right\rangle$ is a cubic KU-subalgebra with an infinite number of different values, which is a contradiction. This completes the proof.

\section{References}


[1] Y.B. Jun, C.S. Kim, M.S. Kang, Cubic subalgebras and ideals of BCK/BCI-algebras, Far East. J. Math. Sci., 44 (2010), 239-250.

[2] S.M. Mostafa, M.A. Abd-Elnaby, M.M. Yousef, Fuzzy ideals of KUalgebras, Int. Math. Forum, 6, No. 63 (2011), 3139-3149.

[3] C. Prabpayak, U. Leerawat, On ideas and congurences in KU-algebras, Scientia Magna J., 5, No. 1 (2009), 54-57.

[4] C. Prabpayak, U. Leerawat, On isomorphisms of KU-algebras, Scientia Magna J., 5, No. 3 (2009), 25-31.

[5] N. Yaqoob, S.M. Mostafa, M.A. Ansari, On cubic KU-ideals of KUalgebras, ISRN Algebra, Article ID 935905 (2013), 10 pages. 
